\title{
THE DUAL RING TO A CORING WITH A GROUPLIKE
}

\author{
MARK KLEINER ${ }^{1}$
}

\begin{abstract}
A matrix problem is that of the classification up to isomorphism of induced comodules over a coring with a grouplike. To define such a problem, using a more familiar language of induced modules, one wants to know what ring is dual to a coring with a grouplike. The answer is given in this paper.
\end{abstract}

We need to recall some definitions from [8].

Let $\Lambda$ be an arbitrary ring with a unit. A $\Lambda$-ring is a $\Lambda$-bimodule $A$ together with two $\Lambda$-bimodule homomorphisms $m: A \otimes_{\Lambda} A \rightarrow A$ and $i: \Lambda \rightarrow A$ such that the following diagrams commute:
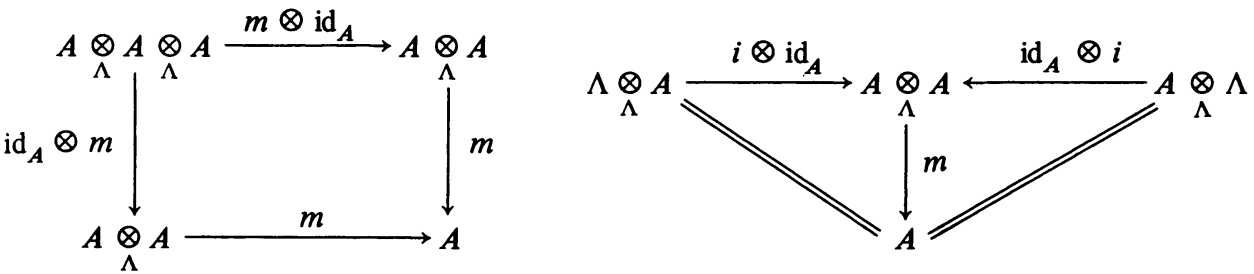

A $\Lambda$-coring is a $\Lambda$-bimodule $C$ together with two $\Lambda$-bimodule homomorphisms $\mu: C \rightarrow C \otimes_{\Lambda} C$ and $\varepsilon: C \rightarrow \Lambda$ which make the dual diagrams commute. An element $g \in C$ is called a grouplike if $\mu(g)=g \otimes g$ and $\varepsilon(g)=1$.

The purpose of this paper is to describe $\Lambda$-rings dual to the corings having a grouplike .

While such rings may be of interest elsewhere, our motivation comes from the theory of matrix problems, i.e. the problems of classifying certain collections of linear maps under the given sets of admissible transformations. A precise definition, various properties, and examples were first given in $[\mathbf{5}, \mathbf{7}]$ using the language of representations of differential graded categories (DGC's). Then it was shown [6] that one can describe every matrix problem by a certain coring, and later the following algebraic formulation was found $[\mathbf{4}]$. A matrix problem is the problem of classification (up to isomorphism) of induced $C$-comodules over a $\Lambda$-coring $C$, i.e. $C$-comodules of the form $C \otimes_{\Lambda} M$, where $M$ belongs to a certain full subcategory of the category of left $\Lambda$-modules.

In one important special case a dual ring to the coring $C$ exists. Let $X^{*}=$ $\operatorname{Hom}_{\Lambda}(X, \Lambda)\left(Y^{0}=\operatorname{Hom}_{\Lambda^{\mathrm{op}}}\left(Y, \Lambda^{\mathrm{op}}\right)\right)$ denote the set of homomorphisms of a right $\Lambda$-module $X$ into $\Lambda$ ( $\Lambda^{\mathrm{op}}$-module $Y$ into $\left.\Lambda^{\mathrm{op}}\right)$. The following statement is proved in $[8$, Proposition 3.2, p. 398 and the Dual Coring Theorem 3.7, p. 403; 4, Corollary 4.2, p. 179].

Received by the editors April 18, 1983.

1980 Mathematics Subject Classification. Primary 16A45; Secondary 16A49.

Key words and phrases. Coring, grouplike, dual ring.

${ }^{1}$ Supported by a Syracuse University Research Grant. 
THEOREM 1. Let $A(C)$ be a $\Lambda$-ring (coring) finitely generated and projective as a right $\Lambda$-module.

(a) $A^{*}\left(C^{*}\right)$ is a $\Lambda^{\mathrm{op}}$-coring (ring) finitely generated and projective as a right $\Lambda^{\mathrm{op}}$-module.

(b) There exist natural isomorphisms $\left(A^{*}\right)^{0} \cong A$ and $\left(C^{*}\right)^{0} \cong C$.

The natural question of whether one can study induced $C^{*}$-modules instead of induced $C$-comodules has a positive answer under some restrictions. Suppose $\Lambda$ is an artin algebra $[1$, p. 257], i.e. an $R$-algebra which is a finitely generated $R$ module, where $R$ is a commutative artin ring with the radical $\underline{r}$. If $I(R / \underline{r})$ is an injective envelope of $R / \underline{r}$, then $D=\operatorname{Hom}_{R}(, I(R / \underline{r}))$ is a duality between $\bmod \Lambda$ and $\bmod \Lambda^{\mathrm{op}}[2, \mathrm{p} .242]$, where $\bmod \Lambda$ is the category of finitely generated $\Lambda$ modules. If $\Lambda$ is an order over a complete discrete valuation ring $R$, then $\bmod \Lambda$ denotes the category of $\Lambda$-lattices, and the duality between $\bmod \Lambda$ and $\bmod \Lambda^{\text {op }}$ is given by $D=\operatorname{Hom}_{R}\left({ }_{-}, R\right)$. For a $\Lambda$-ring (coring) $A(C)$, induc $A$ (induc $C$ ) is the category of induced modules (comodules) of the form $A \otimes_{\Lambda} M\left(C \otimes_{\Lambda} M\right)$, where $M$ belongs to $\bmod \Lambda$. From $[4$, Theorem 4.4, p. 181] we get

THEOREM 2. Let $\Lambda$ be an artin algebra or an order over a complete discrete valuation ring. If $A(C)$ is a $\Lambda$-ring (coring) finitely generated and projective as a right $\Lambda$-module, then $D$ is a duality between the categories induc $A$ and induc $A^{*}$ (induc $C$ and induc $C^{*}$ ).

Theorems 1 and 2 allow us to introduce matrix problems as those of classification of induced modules, without resorting to the language of corings or DGC's. Nevertheless, there is an essential restriction to carry over from that language. All corings corresponding to DGC's have grouplikes, while corings without grouplikes lead to "pathological" problems even in very simple situations [6, p. 308]. The question: What rings are dual to corings with a grouplike? is answered by

THEOREM 3. Let $A$ be a $\Lambda$-ring finitely generated and projective as a right $\Lambda$ module. The dual $\Lambda^{\mathrm{op}}$-coring $A^{*}$ has a grouplike if and only if, as a right module homomorphism, the structure map $i: \Lambda \rightarrow A$ has a left inverse whose kernel is a right ideal of $A$.

Proof. We first show if a $\Lambda$-coring $(C, \mu, \varepsilon)$ has a grouplike $g$, then $C^{*}$ is a $\Lambda^{\mathrm{op}}$-ring satisfying the conditions of Theorem 3. By Theorem 1 , this will prove the necessity. That $C^{*}$ is a $\Lambda^{\text {op }}$-ring follows from [8, Proposition 3.2, p. 398] where the multiplication $m: C^{*} \otimes_{\Lambda^{\circ p}} C^{*} \rightarrow C^{*}$ and unit $i: \Lambda^{\text {op }} \rightarrow C^{*}$ are defined as follows:

$$
m(f \otimes h)(c)=\sum_{i} h\left(f\left(x_{i}\right) y_{i}\right) ; \quad i(\lambda)(c)=\varepsilon(\lambda c)
$$

for all $f, h \in C^{*}, c \in C, \lambda \in \Lambda$, where $\mu(c)=\sum_{i} x_{i} \otimes y_{i}$. Since $i(\lambda)(g)=\varepsilon(\lambda g)=$ $\lambda \varepsilon(g)=\lambda$, evaluation at $g$, i.e. the map $g^{*}: C^{*} \rightarrow \Lambda^{\text {op }}$ with $g^{*}(f)=f(g), f \in C^{*}$, is a left inverse to $i: \Lambda^{\mathrm{op}} \rightarrow C$. It remains to show that $\operatorname{Ker} g^{*}$ is a right $C^{*}$-ideal. But if $f \in \operatorname{Ker} g^{*}$ and $h \in C^{*}$, then $m(f \otimes h)(g)=h(f(g) g)=0$ because $\mu(g)=g \otimes g$. Thus $m(f \otimes h) \in \operatorname{Ker} g^{*}$.

Prove now the sufficiency of Theorem 3. Theorem 1 implies that $A^{*}$ is a $\Lambda^{\text {op }}$ coring. Show $g$ is a grouplike in $A^{*}$ if $g i=1$ and $\operatorname{Ker} g$ is a right ideal. 
Apply the contravariant functor $*=\operatorname{Hom}_{\Lambda}(-, \Lambda)$ to the following diagram of $\Lambda$-bimodule homomorphisms:

$$
A \otimes \stackrel{m}{\longrightarrow} A \stackrel{i}{\longleftarrow} \Lambda
$$

We get the diagram

$$
\left(A \otimes{ }_{\Lambda} A\right)^{*} \stackrel{m^{*}}{\longleftarrow} A^{*} \stackrel{i^{*}}{\longrightarrow} \Lambda^{\mathrm{op}}
$$

of $\Lambda^{\text {op }}$-bimodule homomorphisms (here we identify $\Lambda^{*}$ with $\Lambda^{\mathrm{op}}$ ). Since $A$ is finitely generated and projective as a right $\Lambda$-module, there exists a natural isomorphism $s: A^{*} \otimes_{\Lambda^{\mathrm{op}}} A^{*} \rightarrow\left(A \otimes_{\Lambda} A\right)^{*}$ of $\Lambda^{\mathrm{op}}$-bimodules given by the formula

$$
s(f \otimes h)(x \otimes y)=h(f(x) y) \quad \text { for all } f, h \in A^{*}, x, y \in A ;
$$

see [4, Proposition 4.1, p. 179] or, more generally, [8, Linear Lemma 3.4, p. 400]. From the proof of Theorem 1 [4, pp. 179-180] we see that the $\Lambda^{\text {op }}$-coring structure on $A^{*}$ is given by the comultiplication $\mu=s^{-1} m^{*}$ and counit $\varepsilon=i^{*}$. We have $\varepsilon(g)=i^{*}(g)=g i=1$. To prove that $\mu(g)=s^{-1} m^{*}(g)=g \otimes g$, it suffices to show that $s(g \otimes g)=m^{*}(g)$. From $s(g \otimes g)(x \otimes y)=g(g(x) y), m^{*}(g)(x \otimes y)=g(x y)$, and $g(x) y=(i g(x)) y$, one obtains

$$
\left(m^{*}(g)-s(g \otimes g)\right)(x \otimes y)=g((x-i g(x)) y)=0,
$$

since $x-i g(x) \in \operatorname{Ker} g$ which is a right ideal of $A$. Hence $s(g \otimes g)=m^{*}(g)$. Q.E.D.

REMARKS. 1. Interchanging the words left and right in Theorems $1-3$, one gets valid statements.

2. The rings described in Theorem 3 constitute a special case of augmented rings in the terminology of [3]; see p. 143.

The author is grateful to the referee whose suggestions were very helpful in improving the presentation.

\section{REFERENCES}

1. M. Auslander, Representation theory of artin algebras. I, Comm. Algebra 1 (1974), 177-268.

2. M. Auslander and I. Reiten, Representation theory of artin algebras. III: Almost split sequences, Comm. Algebra 3 (1975), 239-294.

3. H. Cartan and S. Eilenberg, Homological algebra, Princeton Univ. Press, Princeton, N.J., 1956.

4. M. Kleiner, Induced modules and comodules and representations of BOCS's and DGC's, Lecture Notes in Math., vol. 903, Springer-Verlag, Berlin and New York, 1981, pp. 168-185.

5. M. Kleiner and A. V. Roiter, Representations of differential graded categories, Matrix Problems, Kiev, 1977, pp. 5-70. (Russian)

6. A. V. Roiter, Matrix problems and representations of BOCS's, Lecture Notes in Math., vol. 831, Springer-Verlag, Berlin and New York, 1980, pp. 288-324.

7. A. V. Roiter and M. Kleiner, Representations of differential graded categories, Lecture Notes in Math., vol. 488, Springer-Verlag, Berlin and New York, 1975, pp. 316-339.

8. M. E. Sweedler, The predual theorem to the Jacobson-Bourbaki theorem, Trans. Amer. Math. Soc. 213 (1975), 391-406.

Department of Mathematics, Syracuse University, Syracuse, New York 